\title{
Evaluation of the relationship of cholinergic metabolites in urine and urgency urinary incontinence
}

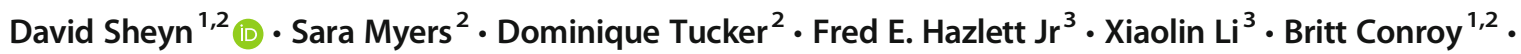 \\ Adonis K. Hijaz ${ }^{1,2}$
}

Received: 4 December 2020 / Accepted: 24 March 2021 / Published online: 5 April 2021

(C) The International Urogynecological Association 2021

\begin{abstract}
Introduction and hypothesis To investigate differences in urine cholinergic metabolites in women with urinary urgency incontinence (UUI) and responders (R) and non-responders (NR) to anti-cholinergic medications (AC).

Methods Patients with UUI and age-matched controls were evaluated pre- and post-treatment using OABSS, UDI-6 and IIQ-7. Controls were defined as having a cumulative OABSS of zero. Patients with UUI were treated with AC and followed for 12 weeks. Responders were those with a $>50 \%$ decrease in the total OABSS score. Urine samples were collected from all participants for evaluation. Metabolite detection was accomplished using commercial assay kits. Wilcoxon-rank sum test and Fisher's exact test were used to express differences between groups. Spearman's rho correlation coefficient was used to determine the relationship between acetylcholine (Ach), choline (Ch), acetylcholinesterase (AchE) and questionnaire scores.

Results We recruited 39 with UUI and 33 controls. We found concentrations of Ch [29.0 (IQR: 24.2-42.5) $\mu$ mol vs. 15.2 (IQR: 7.5-24.1) $\mu \mathrm{mol}$ ] and Ach [65.8 (IQR: 30.4-101.8) nmol and 33.1 (IQR: 11.9-43.8) nmol] were higher in the UUI group compared to controls $(p=0.003$ and $p<0.001$, respectively] and no differences in AchE concentrations. In the UUI group, 43.6\% responded to AC after 12 weeks of therapy. There were no differences in Ch or AchE levels between R and NR; Ach levels were higher in the R group [82.1 nmol (IQR: 54.8-118.1) vs. $50.3 \mathrm{nmol}$ (IQR: 29.9-68.2), $p=0.007$ ]. Ch and Ach were positively associated with pre-treatment OABSS parameters.

Conclusions Urine Ach is higher in responders to anti-cholinergic therapy, and urine cholinergic metabolites were higher in the UUI patients compared to controls.
\end{abstract}

Keywords Urgency incontinence $\cdot$ Choline $\cdot$ Acetylcholine

\section{Introduction}

Overactive bladder with urinary urgency incontinence (UUI) is estimated to affect millions of women globally; in a national survey of 5000 households, $9.3 \%$ of all women and $19.1 \%$ of

David Sheyn

david.sheyn@uhhospitals.org

1 Division of Female Pelvic Medicine and Reconstructive Surgery, Department of Urology, University Hospitals Cleveland Medical Center, 11000 Euclid Avenue, Cleveland, OH 44106, USA

2 Case Western Reserve University School of Medicine, Cleveland, $\mathrm{OH}$, USA

3 Department of Nutrition, Case Western Reserve University, Cleveland, OH, USA those older than 65 years complained of UUI [1]. Women aged 60 to 80 years are among the fastest growing segment of the female population, and thus the prevalence and economic burden of UUI, estimated at nearly $\$ 25$ billion annually, is projected to rapidly increase over time [2,3]. Additionally, not only does UUI have a significant negative impact on quality of life (QOL), but it is also associated with a higher burden and prevalence of anxiety and depression compared to similar groups without lower urinary tract symptoms [4].

While some patients with UUI have an identifiable underlying cause such as neurodegenerative disease, dementia, pelvic floor prolapse, outlet obstruction and medications, in the majority of cases its etiology is unknown and classified as "idiopathic" [5]. However, accumulating evidence now suggests that idiopathic UUI is likely due to sub-clinical dysfunction of a variety of organ systems including the central, peripheral and autonomic nervous systems, cardiovascular 
system, endocrine system and musculoskeletal system [6-9]. However, regardless of the underlying causes, the same UUI care pathway consisting of behavioral modification, pelvic floor physical therapy, pharmacotherapy and third-line therapy is universally applied to patients presenting with UUI because of the difficulty of discerning the dominant cause for their complaints $[10,11]$. This often leads to patient frustration and attrition [12].

As a result, increased attention is being focused on how to best identify the most suitable treatment for individual patients using a patient-centered rather than a "one size fits all" approach. Researchers are exploring utilizing specific biomarkers including interleukins, tumor necrosis factor-alpha and nerve growth factor to guide therapeutic intervention; however, none of these biomarkers consider the fact that the primary pharmacologic mediators of $\mathrm{OAB}$ are anticholinergic medications [13]. Previously, our group investigated the relationship between urinary choline $(\mathrm{Ch})$ and acetylcholine (Ach) in patients with and without UUI as well as in patients who responded to anti-cholinergic therapy versus those who did not. In these studies we found that concentrations of urinary $\mathrm{Ch}$ differed between patients with and without UUI but not between responders and non-responders and that patients who responded to anti-cholinergic therapy had higher levels of pre-treatment Ach in their urine [14, 15]. While our previous studies demonstrated statistically significant findings, they had several limitations, including the inability to assess urinary Ach levels in patients without UUI because of the lack of sensitive assay, small sample size and no evaluation of acetylcholinesterase (AchE) activity. In this study we aimed to measure urinary Ach, $\mathrm{Ch}$ and AchE concentration differences in a larger group of patients, with and without UUI, as well as differences in these urinary markers in responders and non-responders to anti-cholinergic therapy.

\section{Materials and methods}

This was a prospective observational cohort study conducted between September 2019 and April 2020, approved by the Institutional Review Board (IRB\# 01-17-27). Strengthening the Reporting of Observational Studies in Epidemiology (STROBE) guidelines were adhered to in the preparation of this manuscript [16].

Patients presenting to female pelvic medicine and reconstructive surgery (FPMRS) clinics for UUI were recruited for study participation if they met the following inclusion criteria: were 18 years or older, with symptoms of urgency incontinence for a minimum of 3 months and were eligible for treatment with anticholinergic medications with no prior history of sacral or posterior tibial neuromodulation and/or intradetrusor botulinum toxin injection for $\mathrm{OAB}$, current behavioral and/or formal pelvic floor physical therapy, or undergoing treatment with anti-cholinergic or beta-3-agonists within 4 weeks of recruitment and had an OABSS score $>0$. Patients with mixed-urinary incontinence (MUI) were included if their urgency incontinence was the dominant complaint based on patient report, and patients with stress-dominant MUI were excluded. Patients performing self-directed Kegel exercises were allowed to participate. The control group included women presenting to FPMRS clinics for complaints related to pelvic organ prolapse or stress incontinence, annual follow-up after pelvic reconstructive surgery and annual well woman examinations and who had a total OABSS score $=0$.

Any women in the $\mathrm{OAB}$ or control group meeting the following criteria were excluded from the analysis: pregnant within 12 months of study recruitment; currently breast feeding; post-void residual > $200 \mathrm{ml}$; a history of bladder augmentation, anti-incontinence surgery or any acquired or congenital neurologic disorder, except for migrainous and nonmigrainous headaches, including but not limited to neural tube defects, multiple sclerosis and other demyelinating disorders, Parkinson's disease, stroke, brain tumor, traumatic spinal cord or brain injury, myasthenia gravis and diabetes mellitus; also excluded were those being treated with non-bladder-specific medication that had anticholinergic properties, a diuretic, local or systemic estrogen or selective estrogen receptor modulators; women who had a history of liver disease, alcohol abuse, narrow-angle glaucoma, clinical diagnosis of chronic kidney disease or serum creatinine $>1.5 \mathrm{mg} / \mathrm{dl}$; women who were currently being evaluated or treated for recurrent urinary tract infections, interstitial cystitis or painful bladder syndrome defined as the presence of pelvic or suprapubic pain or discomfort associated with irritative voiding symptoms for $>6$ weeks, chronic pelvic pain and/or dyspareunia or those who had a history of genitourinary malignancy. Patients were also excluded if urinalysis data were concerning for urinary tract infection and/or demonstrated hematuria.

If patients met all exclusion and inclusion criteria, they were invited to participate in the study, and, if they agreed, written informed consent was obtained. The criteria used to include patients in the study were reviewed by the principal investigator (DS) prior to inclusion in the final analysis to ensure that uniform methods were used to obtain the UUI and control groups. Demographic and clinical characteristics were collected for each participant including: age, BMI, parity menopausal status, pelvic surgery history and smoking status from the electronic medical record and by history. All patients were asked to complete the Overactive Bladder Symptom Score (OABSS), the Urogenital Distress Inventory-6 (UDI6) and Incontinence Impact Questionnaire-7 (IIQ-7) [17, 18]. The OABSS score ranges from 0 to 15 , and UDI-6 and IIQ-7 range from 0 to 100 , with higher scores indicating greater symptom severity. All patients underwent standard pelvic examination as part of the assessment of their presenting complaint, which included pelvic organ prolapse quantification 
(POP-Q) while in semi-recumbent position and with Valsalva maneuver. Patients also underwent evaluation of a post-void residual by ultrasound to exclude urinary retention.

Patients were initiated on the maximum dose of an anticholinergic and treated for approximately 12 weeks. They were contacted by telephone and were again asked to complete the IIQ-7, UDI-6 and OABSS questionnaires. Treatment success was defined as at least $>50 \%$ decrease in the total OABSS score [19]. The original study protocol was designed for patients to come to the clinic to submit a post-treatment urine sample; however, with the onset of the coronavirus pandemic many patients voiced concern about office visits, and shortly before the final group of patients was scheduled to submit samples, elective in-person clinic visits were temporarily shut down. As a result, there were insufficient samples to conduct a post-treatment urinary analysis.

\section{Sample collection, storage and analysis}

The protocol for specimen collection is as follows. Specimens were collected mid-stream into $120-\mathrm{ml}$ containers per routine protocol for urinalysis (UA) in the office and were evaluated for blood, nitrites, and moderate or high leukocyte esterase; samples positive for any of these findings were excluded from analysis. To account for differences in urinary dilution, samples that were too dilute, had specific gravity $(\mathrm{SG})<1.015$, were too concentrated or had SG $>1.030$ were excluded from the study [20]. Samples were stored in a freezer in the clinic at $-20{ }^{\circ} \mathrm{C}$ for a period of 4 to $6 \mathrm{~h}$, transported to the proteomics laboratory and stored at $-80^{\circ} \mathrm{C}$. Transit time from the clinic site to the laboratory was $<30 \mathrm{~min}$.

The method for evaluating urinary Ach and Ch was described in a previous paper [15]. Acetylcholinesterase (AchE) levels were measured using an ELISA kit (Human ACHEELISA kit, Aviva Systems Biology) and following the manufacturer's protocol. All incubation steps were carried out at $37{ }^{\circ} \mathrm{C}$. Briefly, urine samples were thawed and centrifuged at $2000 \times \mathrm{g}$ for $5 \mathrm{~min}$, and $100 \mu \mathrm{l}$ was added in duplicate along with a standard curve to microtiter plate wells pre-coated with capture antibody. They were then incubated for $2 \mathrm{~h}$. Liquid was removed and $100 \mu$ of biotinylated detector antibody added, followed by incubation for $1 \mathrm{~h}$. Liquid was again removed and the plate washed three times; $100 \mu$ l avidin-horseradish peroxidase conjugate was added to the wells and incubated for $1 \mathrm{~h}$. After five more plate washes, $90 \mu \mathrm{l}$ of TMB substrate was added and the plate incubated in the dark for 15-30 min until the wells turned gradations of blue. Color development was stopped and changed to yellow by adding $50 \mu \mathrm{l}$ of stop solution. O.D. absorbance at $450 \mathrm{~nm}$ was read within $5 \mathrm{~min}$ on a microplate reader (accuSkan FC, Fisher Scientific). AchE was quantified by comparing sample absorbance at $450 \mathrm{~nm}$ with that of the standard curve using microplate reader software (SkanIt RE 4.0, Thermo Scientific).

\section{Statistical analysis}

Based on our previous studies, we determined that we would need 14 patients in the responder and non-responder groups to detect statistically significant $(p<0.05)$ differences in acetylcholine levels with $80 \%$ power, using a median $83.1 \mathrm{nmol}$ acetylcholine concentration in the responder group and assuming a minimum of $50 \%$ lower Ach concentration in the non-responder group $[14,15]$. To account for patient attrition and given a 40-50\% treatment failure rate, we determined we would need to recruit a total of 40 patients with urgency incontinence $[21,22]$. These were matched to patients without urgency incontinence by age. Groups were compared first by presence or absence of UUI, and a sub-group analysis of responders and non-responders was performed. Descriptive statistics are expressed as medians and interquartile ranges. Pairwise analysis using Wilcoxon rank sum test for continuous variables and Fisher's exact test for categorical variables were used as appropriate to express differences between groups with statistical significance set at $p<0.05$. Spearman's rho correlation coefficient was used to determine the relationship between Ach, AchE and Ch concentrations and pre- and post-treatment OABSS scores for daytime frequency, urgency incontinence episodes, nocturia episodes, total OABSS score, UDI-6 and IIQ-7 scores as well as age, BMI and parity. All statistical analysis was performed using STATA version 14.1 (Stata Corp, College Station, TX).

This publication was made possible by the Clinical and Translational Science Collaborative of Cleveland, UL1TR002548, from the National Center for Advancing Translational Sciences (NCATS) component of the National Institutes of Health and NIH Roadmap for Medical Research. Its contents are solely the responsibility of the authors and do not necessarily represent the official views of the NIH.

\section{Results}

Eighty-nine women were screened during the study period and 72 were enrolled in the study, 39 with urgency incontinence and 33 controls. The most common reason for exclusion was opting for therapy with mirabegron or behavioral modification only; additional reasons for exclusion included abnormal UA findings, previous therapy with third-line modalities for OAB or current diuretic use (Fig. 1).

Patients with UUI were similar in age to controls (63 IQR: 52-74 and 64 IQR: 52-77, $p=0.96$, respectively) and did not differ on the basis of race, smoking status, comorbid stress incontinence, post-void residual and prior pelvic floor surgery (Table 1). Patients with UUI had higher BMIs than controls, 33.8 (IQR: 29.2-37.8) kg/m² versus 28.5 (IQR: 25.8-31.2) $\mathrm{kg} / \mathrm{m}^{2}, p=0.006$; had fewer children, $p=0.001$ and were less likely to have anterior wall and apical descent $(p=0.001$ and 


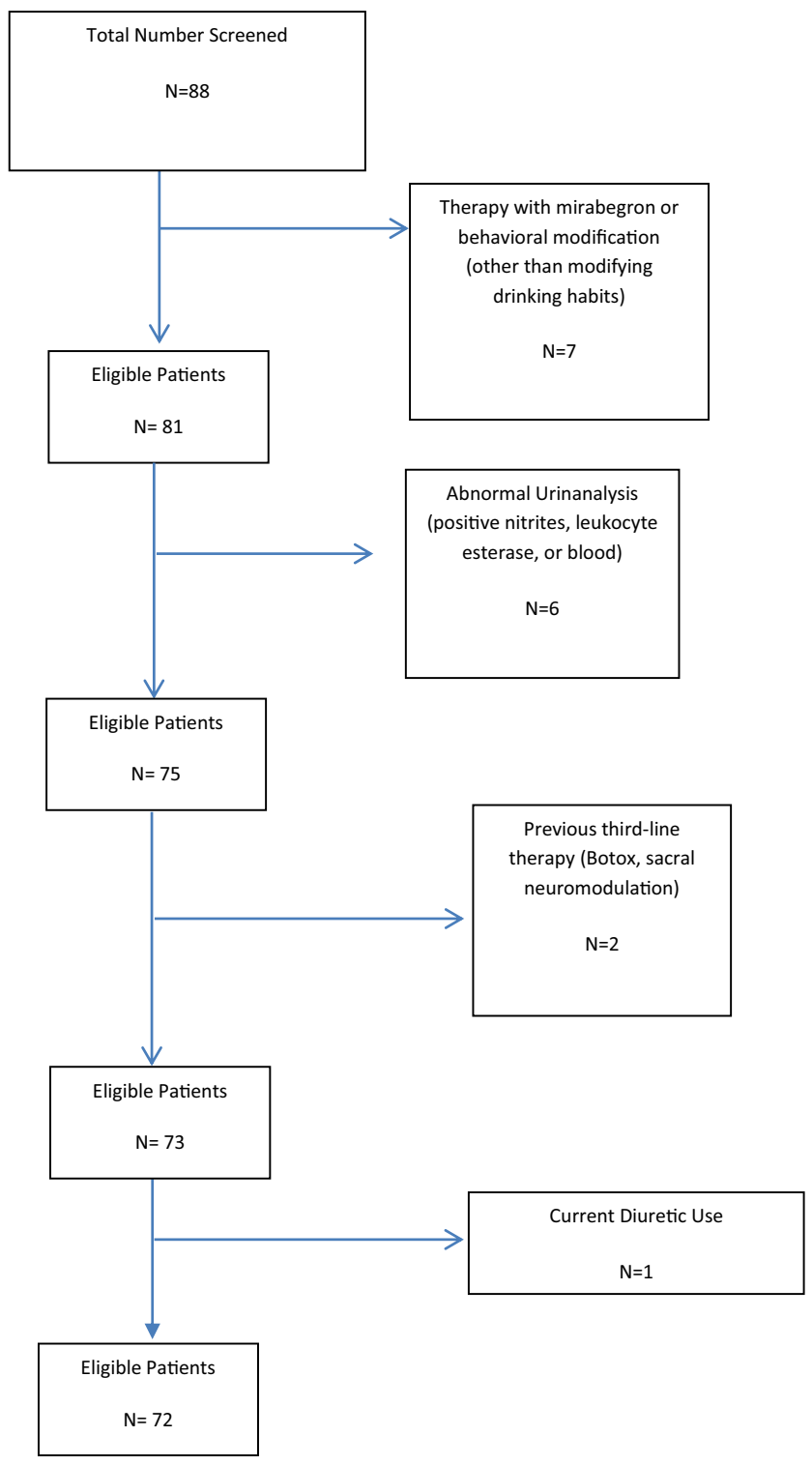

Fig. 1 Flow chart of patient inclusion and exclusion criteria

$p=0.008$, respectively). For patients with UUI the median pre-treatment OABSS score was 11 (IQR: 9-12), the median pre-treatment UDI-6 score was 47.9 (IQR: 37.5-58.0), and the median pre-treatment IIQ-7 score was 60.2 (IQR: 42.8-71.5). The concentrations of Ch 29.0 [(IQR: 24.2-42.5) $\mu \mathrm{mol}$ versus 15.2 (IQR: 7.5-24.1) $\mu \mathrm{mol}$ ] and acetylcholine 65.8 [IQR: 30.4-101.8) nmol and 33.1 (IQR: 11.9-43.8) nmol] were significantly higher in the UUI group compared to controls $(p=$ 0.003 and $p<0.001$, respectively).

Of the 39 patients with UUI, $43.6 \%(n=17)$ had a $>50 \%$ decrease in OABSS scores after 12 weeks of therapy, and the remainder did not. The responder group had lower BMI, $29.5 \mathrm{~kg} / \mathrm{m}^{2}$ (IQR: 25.1-33.9), compared to non-responders, $36.0 \mathrm{~kg} / \mathrm{m}^{2}$ (IQR: $32.1-42.9$ ), $p=0.03$, Table 2. The groups did not differ in age, parity, smoking history, comorbid stress incontinence, surgical history or POP-Q. The responder group also had higher pre-treatment nocturia scores, with a median three or more episodes per night, versus a median of two episodes per night for non-responders. Both groups had similar pre-treatment OABSS, UDI-6 and IIQ-7 scores. Three (17.6\%) patients in the responder group and four (18.2\%) in the responder group were previously treated with an anti-cholinergic for $\mathrm{OAB}$; the median number of medications tried prior to study inclusion was 0 (IQR: 0 0 ) in both groups. The most common medication used for treatment of UUI in both groups was oxybutynin, accounting for $66.9 \%$, and the remainder were prescribed trospium. The median number of medications trialed prior to initiation of anti-cholinergic therapy was 0 (IQR: $0-0$ ).

The median time to follow-up was approximately 90 days for both groups, $p=0.65$; the median post-treatment OABBS score in the responder group was 4 (IQR: 2-5) and 11 (IQR: $9-12)$ in the non-responder group, $p<0.001$. The median post-treatment UDI-6 score was 30 (IQR: 17-52) and 50 (IQR: 42-58), $p=0.002$, in the responder and non-responder groups, respectively. The median post-treatment IIQ-7 scores were 49 (IQR: 36-61) in the responder group and 65 (IQR: 48-76) in the non-responder group, $p=0.01$. There was no difference in Ch $(p=0.38)$ or $\operatorname{AchE}(p=0.85)$ between groups; acetylcholine levels were higher in the responder group, $82.1 \mathrm{nmol}$ (IQR: 54.8-118.1), compared to the nonresponder group, $50.3 \mathrm{nmol}$ (IQR: 29.9-68.2).

Table 3 demonstrates the clinical characteristics and urine metabolite levels in the control group compared to responders and non-responders. Both responders and non-responders had fewer children compared to controls, $p=0.01$ and $p=0.02$, respectively. Non-responders had significantly higher BMI compared to controls, $36.0 \mathrm{~kg} / \mathrm{m}^{2}$ versus $28.5 \mathrm{~kg} / \mathrm{m}^{2}$ with $p=0=$ 0.001, while BMI was similar between controls and responders. The responder and non-responder groups both were more likely not to have anterior or apical prolapse compared to the control group, where the median value of Aa was -1 and $\mathrm{C}$ was -6 . The $\mathrm{Ch}$ and Ach levels in responders and non-responders were significantly higher than in the control group.

The relationships between Ach and Ch levels and patient characteristics are shown in Table 4. Age was not found to be associated with levels of either metabolite; however, BMI had a weak positive correlation with choline, Spearman $\rho=0.369$, $p=0.001$. Both choline and acetylcholine demonstrated significant but predominantly weak positive associations with pre-treatment frequency, urgency, UUI and nocturia scores as well as the total OABSS score. The strongest of these associations was between acetylcholine and pre-treatment urgency, Spearman $\rho=0.426, p=0.03$.

\section{Discussion}

In this prospective cohort study, we found that women with UUI have significantly higher levels of choline and 
Table 1 Patient characteristics stratified by presence of urgency urinary incontinence

\begin{tabular}{|c|c|c|c|}
\hline & $\begin{array}{l}\text { Urgency incontinence } \\
N=39\end{array}$ & $\begin{array}{l}\text { No urgency incontinence } \\
N=33\end{array}$ & $P$ \\
\hline Age (years), median (IQR*) & $63(52-74)$ & $64(52-77)$ & 0.96 \\
\hline Body mass index $\left(\mathrm{kg} / \mathrm{m}^{2}\right)$, median (IQR) & $33.8(29.2-37.8)$ & $28.5(25.8-31.2)$ & 0.006 \\
\hline Parity, median (IQR) & $2(1-2)$ & $3(2-4)$ & 0.001 \\
\hline Non-white race & $11(28.2)$ & $15(45.5)$ & 0.05 \\
\hline Current smoker & $8(20.5)$ & $6(18.2)$ & 0.52 \\
\hline Presence of stress urinary incontinence & $22(56.4)$ & $16(48.5)$ & 0.86 \\
\hline Constipation & $4(10.2)$ & $3(9.1)$ & 0.62 \\
\hline Post-void residual (ml), median (IQR) & $34(0-69)$ & $15(0-44)$ & 0.17 \\
\hline History of hysterectomy & $10(25.6)$ & $11(33.3)$ & 0.89 \\
\hline $\begin{array}{l}\text { Prior history of pelvic organ prolapse repair } \\
\text { Apical }\end{array}$ & $\begin{array}{l}5(12.8) \\
2(5.1)\end{array}$ & $\begin{array}{l}9(27.3) \\
1(3.0)\end{array}$ & 0.70 \\
\hline Anterior & $2(5.1)$ & $5(15.2)$ & \\
\hline Posterior & $1(2.6)$ & $3(9.1)$ & \\
\hline Post-menopausal status & $28(71.8)$ & $25(75.8)$ & 0.91 \\
\hline Aa, median (IQR) & $-3(-3--2)$ & $-1(-3-1)$ & 0.001 \\
\hline $\mathrm{C}$, median (IQR) & $-8(-8-6)$ & $-6(-8--3)$ & 0.008 \\
\hline Pretreatment OABSS frequency score (range $0-2$ ), median (IQR) & $1(0-2)$ & 0 & - \\
\hline Pretreatment OABSS Nocturia Score (range 0-3), median (IQR) & $3(2-3)$ & 0 & - \\
\hline Pretreatment OABSS Urgency Score (range 0-5), median (IQR) & $4(3-5)$ & 0 & - \\
\hline Pretreatment OABSS Urgency Incontinence Score (range 0-5), median (IQR) & $3(2-4)$ & 0 & - \\
\hline Pretreatment OABSS Total (range 0-15), median (IQR) & $11(9-12)$ & 0 & - \\
\hline Pretreatment Urogenital Distress Inventory-6 Score (range 0-100), median (IQR) & $47.9(37.5-58)$ & - & - \\
\hline Pretreatment Incontinence Impact Questionnaire-7 Score (range 0-100), median (IQR) & $60.2(42.8-71.5)$ & - & - \\
\hline Pretreatment Urine Specific Gravity, median (IQR) & $1.015(1.015-1.020)$ & $1.020(1.015-1.020)$ & 0.79 \\
\hline Pretreatment choline (micromoles), median (IQR) & $29.0(24.2-42.5)$ & $15.2(7.5-24.1)$ & 0.003 \\
\hline Pretreatment acetylcholine (nanomoles), median (IQR) & $65.8(30.4-101.8)$ & $33.1(11.9-43.8)$ & $<0.001$ \\
\hline Pretreatment acetylcholinesterase ( $\mathrm{ng} / \mathrm{ml})$, median (IQR) & $0.14(0.06-0.40)$ & $0.18(0.05-0.39)$ & 0.90 \\
\hline
\end{tabular}

Values expressed as median (interquartile range) or $\mathrm{N}(\%)$

*IQR: interquartile Range

acetylcholine in their urine compared to women without any urgency symptoms and that women with UUI who respond to anti-cholinergic therapy, based on a $>50 \%$ decrease in OABSS scores, have higher levels of urinary acetylcholine compared to women who do not respond. No differences in acetylcholinesterase activity were noted between any of the groups. The results build on our previous work on evaluation of cholinergic urinary metabolites in women with and without UUI and in responders and non-responders [14, 15].

In the prior study evaluating these metabolites in women with and without UUI, acetylcholine could not be measured because of lack of a sensitive assay for measuring the acetylcholine levels; since then, an essay has been acquired by our laboratory with increased sensitivity to Ach allowing these measurements. In this study, Ach levels were found to be higher among women with UUI, as were Ch levels, which differs from our prior results despite using an identical measurement technique in both studies [15]. The difference in Ch levels between the two studies may be related to differences in the demographics of women, with women in this study being a median of 10 years older and more likely to be post-menopausal. Indeed, there is a negative correlation between $\mathrm{Ch}$ and age, indicating that levels may decrease with age; however, this would need to be formally evaluated in a much larger patient cohort, and this has not been previously investigated.

Acetylcholine is the primary neurotransmitter involved in normal and dysfunctional detrusor contraction, and neuronal acetylcholine has classically been associated with OAB [23]. However, there is increasing evidence that the urothelium is a metabolically active region of the bladder, capable of producing its own non-neuronal acetylcholine and has been implicated both in dysfunction detrusor contraction and as a target of anti-cholinergic medications $[24,25]$. While in this study we 
Table 2 Comparison of patients with urgency urinary incontinence who did and did not respond to anti-cholinergic therapy

\begin{tabular}{|c|c|c|c|}
\hline & Responder $N=17$ & Non-responder $N=22$ & $P$ \\
\hline Age (years), median (IQR) & $63(55-79)$ & $57(47-72)$ & 0.21 \\
\hline Body mass index $\left(\mathrm{kg} / \mathrm{m}^{2}\right)$, median (IQR) & $29.9(25.1-33.9)$ & $36.0(32.1-42.9)$ & 0.03 \\
\hline Parity, median (IQR) & $2(1-3)$ & $2(1-2)$ & 0.98 \\
\hline Non-white race & $5(29.4)$ & $6(27.2)$ & 0.43 \\
\hline Current smoker & $3(17.6)$ & $5(22.7)$ & 0.51 \\
\hline Presence of stress urinary incontinence & $12(70.6)$ & $10(45.4)$ & 0.11 \\
\hline Constipation & $3(17.6)$ & $1(4.5)$ & 0.21 \\
\hline Post-void residual (ml), median (IQR) & $17(0-37)$ & $50(14-76)$ & 0.07 \\
\hline History of hysterectomy & $5(29.4)$ & $5(22.7)$ & 0.45 \\
\hline $\begin{array}{l}\text { Prior history of pelvic organ prolapse repair } \\
\text { Apical }\end{array}$ & $\begin{array}{l}2(11.8) \\
1(5.9)\end{array}$ & $\begin{array}{l}3(13.6) \\
1(4.5)\end{array}$ & 0.69 \\
\hline Anterior & $1(5.9)$ & $1(4.5)$ & \\
\hline Posterior & $0(0.0)$ & $1(4.5)$ & \\
\hline Post-menopausal status & $13(76.4)$ & $15(68.2)$ & 0.30 \\
\hline Aa, median (IQR) & $-3(-3--1)$ & $-3(-3--1)$ & 0.49 \\
\hline $\mathrm{C}$, median (IQR) & $-8(-8--6)$ & $-8(-8--8)$ & 0.35 \\
\hline Number of prior medications trialed & $0(0-0)$ & $0(0-0)$ & 0.92 \\
\hline \multicolumn{4}{|l|}{ Medication trialed } \\
\hline Oxybutynin & $12(70.1)$ & $14(63.6)$ & 0.21 \\
\hline Trospium & $5(29.4)$ & $6(27.2)$ & 0.45 \\
\hline Pretreatment OABSS Frequency Score (range 0-2), median (IQR) & $1(1-2)$ & $1(0-1)$ & 0.23 \\
\hline Pretreatment OABSS Nocturia Score (range 0-3), median (IQR) & $3(3-3)$ & $2(2-3)$ & 0.03 \\
\hline Pretreatment OABSS Urgency Score (range 0-5), median (IQR) & $4(4-5)$ & $4(3-4)$ & 0.79 \\
\hline Pretreatment OABSS Urgency Incontinence Score (range 0-5), median (IQR) & $3(2-4)$ & $2(1-4)$ & 0.40 \\
\hline Pretreatment OABSS Total (range $0-15)$, median (IQR) & $11(9-11)$ & $12(9-13)$ & 0.15 \\
\hline Pretreatment Urogenital Distress Inventory-6 Score (range 0-100), median (IQR) & $56(42-67)$ & $50(42-58)$ & 0.09 \\
\hline Pretreatment Incontinence Impact Questionnaire-7 Score (range 0-100), median (IQR) & $67(54-75)$ & $69(48-81)$ & 0.13 \\
\hline Posttreatment OABSS Frequency Score (range $0-2$ ), median (IQR) & $0(0-0)$ & $1(1-2)$ & 0.006 \\
\hline Posttreatment OABSS Nocturia Score (range 0-3), median (IQR) & $1(0-2)$ & $2(2-3)$ & 0.001 \\
\hline Posttreatment OABSS Urgency Score (range 0-5), median (IQR) & $2(1-2)$ & $3(2-4)$ & 0.001 \\
\hline Posttreatment OABSS Urgency Incontinence Score (range 0-5), median (IQR) & $1(0-1)$ & $2(2-4)$ & $<0.001$ \\
\hline Posttreatment OABSS Total (range $0-15)$, median (IQR) & $4(2-5)$ & $11(9-12)$ & $<0.001$ \\
\hline Posttreatment Urogenital Distress Inventory-6 Score (range 0-100), median (IQR) & $30(17-52)$ & $44(33-51)$ & 0.002 \\
\hline Posttreatment Incontinence Impact Questionnaire-7 Score (range 0-100), median (IQR) & $49(36-61)$ & $65(48-76)$ & 0.01 \\
\hline Duration of therapy (days), median (IQR) & $89(84-93)$ & $91(84-96)$ & 0.65 \\
\hline Pretreatment urine specific gravity, median (IQR) & $1.020(1.015-1.020)$ & $1.020(1.015-1.020)$ & 0.93 \\
\hline Pretreatment choline (micromoles), median (IQR) & $37.8(14.3-54.1)$ & $27.0(21.7-36.2)$ & 0.38 \\
\hline Pretreatment acetylcholine (nanomoles), median (IQR) & $82.1(54.8-118.1)$ & $50.3(29.9-68.2)$ & 0.04 \\
\hline Pretreatment acetylcholinesterase (ng/ml), median (IQR) & $0.13(0.08-0.21)$ & $0.13(0.03-0.40)$ & 0.85 \\
\hline
\end{tabular}

Values expressed as median (interquartile range) or $\mathrm{N}(\%)$

were unable to ascertain whether the acetylcholine measured is neuronal or non-neuronal, the acetylcholine levels were directly related to choline levels, which were elevated in both UUI relative to controls and in responders relative to nonresponders. Choline is an essential nutrient and the primary precursor molecule of acetylcholine, and it is not excreted into urine by the kidneys. Thus, the choline measured in this and our prior studies may be directly related to choline being utilized by the urothelium [26, 27].

Additionally, there is an emerging link between the microbiome and urinary choline and acetylcholine. Several studies have identified differences in the urinary microbiome and in women with and without UUI as well as those who do and do not respond to medications. Specifically several 
Table 3 Comparison of clinical characteristics and urine metabolites of responders and non-responders relative to patients without urgency incontinence

\begin{tabular}{|c|c|c|c|c|c|}
\hline & $\begin{array}{l}\text { No urgency incontinence } \\
\mathrm{N}=33\end{array}$ & $\begin{array}{l}\text { Non-responder } \\
\mathrm{N}=22\end{array}$ & $P^{*}$ & $\begin{array}{l}\text { Responder } \\
\mathrm{N}=17\end{array}$ & $p^{!}$ \\
\hline Age (years), median (IQR) & $64(52-77)$ & $57(47-72)$ & 0.32 & $63(55-79)$ & 0.54 \\
\hline Body mass index $\left(\mathrm{kg} / \mathrm{m}^{2}\right)$, median (IQR) & $28.5(25.8-31.2)$ & $36.0(32.1-42.9)$ & 0.001 & $29.9(25.1-33.9)$ & 0.35 \\
\hline Parity, median (IQR) & $3(2-4)$ & $2(1-2)$ & 0.02 & $2(1-3)$ & 0.01 \\
\hline Non-white race & $15(45.5)$ & $6(27.2)$ & 0.18 & $5(29.4)$ & 0.25 \\
\hline Current smoker & $6(18.2)$ & $5(22.7)$ & 0.68 & $3(17.6)$ & 0.92 \\
\hline Presence of stress urinary incontinence & $16(48.5)$ & $10(45.4)$ & 0.82 & $12(70.6)$ & 0.17 \\
\hline Constipation & $3(9.1)$ & $1(4.5)$ & 0.52 & $3(17.6)$ & 0.41 \\
\hline Post-void residual (ml), median (IQR) & $15(0-44)$ & $50(14-76)$ & 0.06 & $17(0-37)$ & 0.91 \\
\hline History of hysterectomy & $11(33.3)$ & $5(22.7)$ & 0.41 & $5(29.4)$ & 0.89 \\
\hline Prior history of pelvic organ prolapse repair & $9(27.3)$ & $3(13.6)$ & 0.64 & $2(11.8)$ & 0.58 \\
\hline Apical & $1(3.0)$ & $1(4.5)$ & & $1(5.9)$ & \\
\hline Anterior & $5(15.2)$ & $1(4.5)$ & & $1(5.9)$ & \\
\hline Posterior & $3(9.1)$ & $1(4.5)$ & & $0(0.0)$ & \\
\hline Post-menopausal status & $25(75.8)$ & $15(68.2)$ & 0.54 & $13(76.4)$ & 0.63 \\
\hline Aa, median (IQR) & $-1(-3-1)$ & $-3(-3--1)$ & 0.002 & $-3(-3$ to -1$)$ & 0.01 \\
\hline $\mathrm{C}$, median (IQR) & $-6(-8--3)$ & $-8(-8--8)$ & 0.01 & $-8(-8--6)$ & 0.03 \\
\hline Pretreatment urine specific gravity, median (IQR) & $1.020(1.015-1.020)$ & $1.020(1.015-1.020)$ & 0.89 & $1.020(1.015-1.020)$ & 0.92 \\
\hline Pretreatment choline (micromoles), median (IQR) & $15.2(7.5-24.1)$ & $27.0(21.7-36.2)$ & 0.001 & $37.8(14.3-54.1)$ & 0.002 \\
\hline Pretreatment acetylcholine (nanomoles), median (IQR) & $33.1(11.9-43.8)$ & $50.3(29.9-68.2)$ & 0.007 & $82.1(54.8-118.1)$ & $<0.001$ \\
\hline Pretreatment acetylcholinesterase $(\mathrm{ng} / \mathrm{ml})$, median (IQR) & $0.18(0.05-0.39)$ & $0.13(0.03-0.40)$ & 0.85 & $0.13(0.08-0.21)$ & 0.86 \\
\hline
\end{tabular}

Values expressed as median (interquartile range) or $\mathrm{N}(\%)$

*Comparison of patients without urgency incontinence to non-responders

'Comparison of patients without urgency incontinence to responders

studies have found that women with UUI have higher levels of uropathogenic bacteria, in particular Proteus and Aerococcus species, and lower levels of Lactobacillus species without overt evidence of infection [28-30]. Furthermore, there is evidence that some bacterial species which make up the urinary microbiome can synthesize and release neurotransmitters, including Ach [31]. Thus, the link between UUI and the microbiome could be related to bacterial production of cholinergic metabolites, though this concept needs to be further investigated.

The findings of higher acetylcholine levels in responders to anti-cholinergic therapy compared to non-responders may suggest that there is a sub-type of $\mathrm{OAB}$, which is primarily mediated by cholinergic signaling mechanisms and may, at least in part, explain why certain patients have dramatic improvements with anti-cholinergic treatment, whereas others with seemingly identical clinical characteristics do not.

This study has several strengths. First, this is the largest cohort to date to investigate differences in cholinergic urine metabolites in women with and without overactive bladder. Second, we were able to reproduce our results with a different study population demonstrating higher levels of acetylcholine and choline in women who respond to anti-cholinergic medications compared to those who did not. We were also able to link choline and acetylcholine levels to OABSS scores, which have been shown to be predictive of the treatment response to anti-cholinergic medications [32].

The study results should be interpreted with the following limitations in mind. Presently, the impact of age and BMI on urine levels of choline and acetylcholine, both of which are associated with $\mathrm{OAB}$, is not well understood [33,34]. Though we accounted for differences in age with matching,, in this analysis there were significant differences in BMI between controls and patients with UUI and responders and non-responders. BMI demonstrated a weak, but statistically significant correlation with choline, which given the fact that choline is an essential dietary nutrient, may indicate a relationship between obesity, diet and bladder function [25, 26]. Notably BMI and acetylcholine levels were not found to have a correlative relationship, and the BMI levels of responders to anticholinergic and controls were not statistically different and there were no differences in choline concentrations between responders and non-responders, though the sample size was very small, and a significant relationship may be found with 


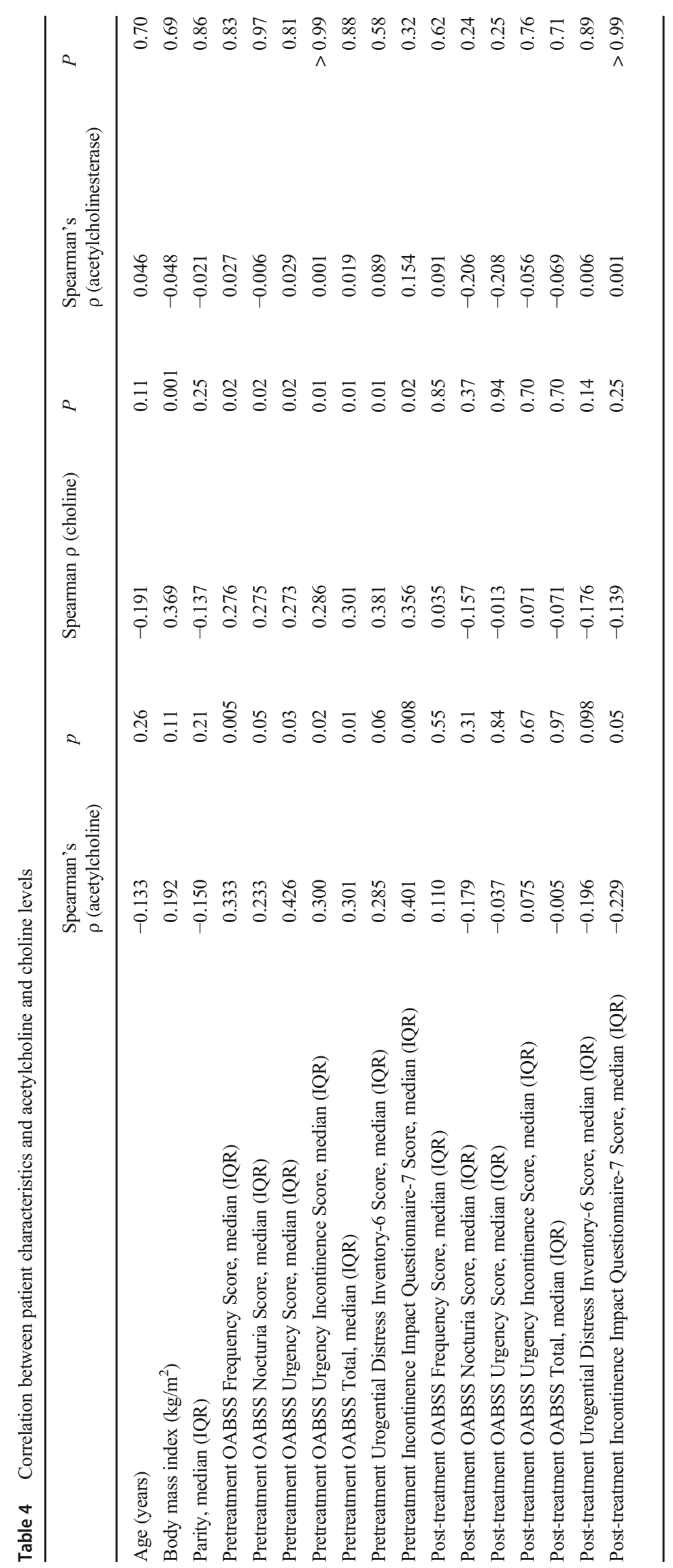


larger studies. Future studies should make an effort to adjust for BMI between groups and to investigate the relationship between BMI, diet, cholinergic metabolites and overactive bladder symptoms. Second, one of the original objectives of this study was to evaluate whether acetylcholine and choline levels changed after treatment with anti-cholinergic therapy and whether this change was statistically different between responders and non-responders; however, due to the COVID-19 pandemic and a moratorium on patient-related research activities, this could not be accomplished. Thus, we are not yet able to determine whether there is a causal relationship between higher choline and acetylcholine levels and anti-cholinergic response. Finally, we are not able to discern whether the choline and acetylcholine levels reflect differential neuronal or urothelial activity, and this would likely require a radio-labeled study to evaluate.

In summary, this study identified higher levels of urinary choline and acetylcholine in patients with UUI compared to controls and higher levels of acetylcholine among responders compared to non-responders. Acetylcholine could potentially serve as a marker for selection of candidates who may respond to anti-cholinergic therapy, while patients with low levels may be prescribed other therapies. However, a further study in larger cohorts and controlling for genetic ancestry and BMI and led by different investigator teams is recommended to both confirm and expand on these findings.

Author contributions DS: Concept, study design, data interpretation, statistical analysis, recruitment, manuscript preparation.

AKH: Concept, study design, recruitment, manuscript preparation.

FEH: data analysis and interpretation, manuscript preparation.

$\mathrm{XL}$ : data analysis and interpretation, manuscript preparation.

$\mathrm{BC}$ : data interpretation, manuscript preparation.

SM: data collection, manuscript preparation, recruitment.

DT: data collection, manuscript preparation, recruitment.

Funding Clinical and Translational Science Collaborative of Cleveland, UL1TR002548 from the National Center for Advancing Translational Sciences (NCATS) component of the National Institutes of Health and NIH Roadmap for Medical Research.

\section{Declarations}

Conflicts of interest None.

\section{References}

1. Stewart WF, Corey R, Herzog AR, et al. Prevalence of overactive bladder in women: results from the NOBLE program. Int Urogynecol J. 2001;12:S66.

2. Lukacz ES, Santiago-Lastra Y, Albo ME, Brubaker L. Urinary incontinence in women: a review. JAMA. 2017;318(16):1592604. https://doi.org/10.1001/jama.2017.12137.

3. Older population and aging. https://www.census.gov/topics/ population/older-aging.html. Accessed October 292020.
4. Coyne KS, Kvasz M, Ireland AM, Milsom I, Kopp ZS, Chapple CR. Urinary incontinence and its relationship to mental health and health-related quality of life in men and women in Sweden, the United Kingdom, and the United States. Eur Urol. 2012;61(1): 88-95. https://doi.org/10.1016/j.eururo.2011.07.049.

5. Peyronnet B, Mironska E, Chapple C, Cardozo L, Oelke M, Dmochowski R, et al. A comprehensive review of overactive bladder pathophysiology: on the way to tailored treatment. Eur Urol. 2019;75(6):988-1000. https://doi.org/10.1016/j.eururo.2019.02. 038.

6. Chan SS, Chan DK, Pang SM, Lam ST, Lao TT, Choy KW. Urinary incontinence should be added to the manifestation in women with Marfan syndrome. Int Urogynecol J. 2010;21(5):583-7. https://doi.org/10.1007/s00192-009-1078-4.

7. Smith AL. Understanding overactive bladder and urgency incontinence: what does the brain have to do with it? F1000Res. 2018;7: F1000 Faculty Rev-1869. https://doi.org/10.12688/f1000research. 16418.1.

8. Speich JE, Tarcan T, Hashitani H, Vahabi B, McCloskey KD, Andersson KE, et al. Are oxidative stress and ischemia significant causes of bladder damage leading to lower urinary tract dysfunction? Report from the ICI-RS 2019. Neurourol Urodyn. 2020;39(Suppl 3):S16-22. https://doi.org/10.1002/nau.24313.

9. Araklitis G, Baines G, da Silva AS, Robinson D, Cardozo L. Recent advances in managing overactive bladder. F1000Res. 2020;9: F1000 Faculty Rev-1125. https://oi.org/10.12688/f1000research. 26607.1.

10. Lightner DJ, Gomelsky A, Souter L, Vasavada SP. Diagnosis and treatment of overactive bladder (non-neurogenic) in adults: AUA/ SUFU guideline amendment 2019. J Urol. 2019;202(3):558-63. https://doi.org/10.1097/JU.0000000000000309.

11. Bedoya-Ronga A, Currie I. Improving the management of urinary incontinence. Practitioner. 2014;258(1769):21-4. 2-3.

12. Rohloff M, Peifer G, Thompson JH. Patient navigation for overactive bladder improves access to care. Int Urogynecol J. 2020;31(5): 1007-12. https://doi.org/10.1007/s00192-019-04085-7.

13. Antunes-Lopes T, Cruz F. Urinary biomarkers in overactive bladder: revisiting the evidence in 2019. Eur Urol Focus. 2019;5(3): 329-36. https://doi.org/10.1016/j.euf.2019.06.006.

14. Sheyn D, Hijaz AK, Hazlett FE Jr, El-Nashar S, Mangel JM, Li X, et al. Evaluation of choline and acetylcholine levels in responders and nonresponders to anticholinergic therapy for overactive bladder syndrome. Female Pelvic Med Reconstr Surg. 2019. https://doi.org/ 10.1097/SPV.0000000000000787.

15. Sheyn D, Hijaz AK, Hazlett FE Jr, Dawodu K, El-Nashar S, Mangel JM, et al. Evaluation of urine choline levels in women with and without overactive bladder syndrome. Female Pelvic Med Reconstr Surg. 2020;26(10):644-8. https://doi.org/10.1097/SPV. 0000000000000639.

16. Von Elm E, Altman DG, Egger M, Pocock SJ, Gøtzsche PC, Vandenbroucke JP. Strobe initiative. The strengthening the reporting of observational studies in epidemiology (STROBE) statement: guidelines for reporting observational studies. Prev Med. 2007;45(4):247-51.

17. Homma Y, Yoshida M, Seki N, Yokoyama O, Kakizaki H, Gotoh $\mathrm{M}$, et al. Symptom assessment tool for overactive bladder syndrome-overactive bladder symptom score. Urology. 2006;68(2):318-23. https://doi.org/10.1016/j.urology.2006.02. 042 .

18. Uebersax JS, Wyman JF, Shumaker SA, McClish DK, Fantl JA. Short forms to assess life quality and symptom distress for urinary incontinence in women: the incontinence impact questionnaire and the urogenital distress inventory. Continence program for women research group. Neurourol Urodyn. 1995;14(2):131-9. https://doi. org/10.1002/nau.1930140206. 
19. Zhao YR, Liu WZ, Guralnick M, Niu WJ, Wang Y, Sun G, et al. Predictors of short-term overactive bladder symptom improvement after transurethral resection of prostate in men with benign prostatic obstruction. Int J Urol. 2014;21(10):1035-40.

20. Edmands WM, Ferrari P, Scalbert A. Normalization to specific gravity prior to analysis improves information recovery from high resolution mass spectrometry metabolomic profiles of human urine. Anal Chem. 2014;86(21):10925-31. https://doi.org/10.1021/ ac503190m.

21. Hsiao SM, Lin HH, Kuo HC. Factors associated with a better therapeutic effect of solifenacin in patients with overactive bladder syndrome. Neurourol Urodyn. 2014;33(3):331-4. https://doi.org/ 10.1002/nau.22394.

22. Wagg A, Compion G, Fahey A, Siddiqui E. Persistence with prescribed antimuscarinic therapy for overactive bladder: a UK experience. BJU Int. 2012;110(11):1767-74. https://doi.org/10.1111/j. 1464-410X.2012.11023.x

23. Yamada S, Ito Y, Nishijima S, Kadekawa K, Sugaya K. Basic and clinical aspects of antimuscarinic agents used to treat overactive bladder. Pharmacol Ther. 2018;189:130-48. https://doi.org/10. 1016/j.pharmthera.2018.04.010.

24. Lips KS, Wunsch J, Zarghooni S, Bschleipfer T, Schukowski K, Weidner W, et al. Acetylcholine and molecular components of its synthesis and release machinery in the urothelium. Eur Urol. 2007;51(4):1042-53. https://doi.org/10.1016/j.eururo.2006.10. 028.

25. Fry $\mathrm{CH}$, Vahabi B. The role of the mucosa in normal and abnormal bladder function. Basic Clin Pharmacol Toxicol. 2016;119(Suppl 3):57-62. https://doi.org/10.1111/bcpt.12626.

26. Zeisel SH, da Costa KA. Choline: an essential nutrient for public health. Nutr Rev. 2009;67(11):615-23. https://doi.org/10.1111/j. 1753-4887.2009.00246.x.

27. Ueland PM. Choline and betaine in health and disease. J Inherit Metab Dis. 2011;34(1):3-15. https://doi.org/10.1007/s10545-0109088-4.
28. Hilt EE, Putonti C, Thomas-White K, Lewis AL, Visick KL, Gilbert NM, et al. Aerococcus urinae isolated from women with lower urinary tract symptoms: In Vitro aggregation and genome analysis. J Bacteriol. 2020;202(13):e00170-20. https://doi.org/10. 1128/JB.00170-20.

29. Wu P, Chen Y, Zhao J, Zhang G, Chen J, Wang J, et al. Urinary microbiome and psychological factors in women with overactive bladder. Front Cell Infect Microbiol. 2017;7:488. https://doi.org/10. 3389/fcimb.2017.00488.

30. Angelini KJ. An integrative review of current research on the role of the female urinary microbiota in overactive bladder symptoms. Urol Nurs. 2017;37(2):94-100.

31. Cryan JF, Dinan TG. Mind-altering microorganisms: the impact of the gut microbiota on brain and behaviour. Nat Rev Neurosci. 2012;13(10):701-12. https://doi.org/10.1038/nrn3346.

32. Hsiao SM, Liao CH, Lin HH, Kuo HC. Duration of antimuscarinic administration for treatment of overactive bladder before which one can assess efficacy: an analysis of predictive factors. Int Neurourol J. 2015;19(3):171-7. https://doi.org/10.5213/inj.2015.19.3.171.

33. Wang Y, Shi C, Wang Y, Jiao W, Wang X, Zhang J, et al. The correlation between severity of overactive bladder symptoms with female sexual dysfunction and sexual satisfaction of partners. Urol Int. 2020;11:1-7. https://doi.org/10.1159/000508764.

34. Irwin DE, Kopp ZS, Agatep B, Milsom I, Abrams P. Worldwide prevalence estimates of lower urinary tract symptoms, overactive bladder, urinary incontinence and bladder outlet obstruction. BJU Int. 2011;108(7):1132-8. https://doi.org/10.1111/j.1464-410X. 2010.09993.x.

Publisher's note Springer Nature remains neutral with regard to jurisdictional claims in published maps and institutional affiliations. 